\title{
Static Stretching at Pain-Tolerated Intensity Is Not Necessary to Increase Knee Range of Motion in Amateur Soccer Players: a Randomized Trial
}

\author{
R. R. V. Melo ${ }^{1}$, M. S. Cerqueira ${ }^{1}$, G. M. Barbosa ${ }^{2}$, A. L. B. A. Laurentino ${ }^{1}$, I. M. Franca ${ }^{1}$, \\ T. O. Souza1, W. H. Brito Vieira ${ }^{1}$ \\ 1 Department of Physical Therapy, Federal University of Rio Grande do Norte, Neuromuscular Performance \\ Analysis Laboratory, Natal, Brazil \\ 2 Faculty of Health Sciences of Trairi, Federal University of Rio Grande do Norte, Santa Cruz, Brazil
}

\author{
CORRESPONDING AUTHOR: \\ Wouber Hérickson Brito Vieira \\ Departamento de Fisioterapia \\ Neuromuscular Performance Analysis \\ Laboratory \\ Universidade Federal do Rio Grande \\ do Norte \\ Avenida Hermes Fonseca \\ 59084-100 Natal, Brazil \\ E-mail: hericksonfisio@yahoo.com.br
}

DOI:

10.32098/mltj.03.2021.19

LEVEL OF EVIDENCE: 2B

\begin{abstract}
Background. Stretching exercises are commonly used in warm-up routines before sports practice. However, there is a lack of evidence regarding the optimal intensity at which passive stretching should be performed in soccer players. This study investigated the acute and chronic effects of different static stretching intensities on the knee ROM, passive torque and functional performance of amateur soccer players.

Methods. Forty-one male amateur soccer players were allocated into four groups: no stretching (NS, $\mathrm{n}=10$ ), comfort level stretching (CLS, $\mathrm{n}=11$ ), mild discomfort level stretching (MDLS, $\mathrm{n}=10$ ) or pain level stretching (PLS, $\mathrm{n}=10$ ). Three 30-second sessions of static passive hamstring muscle stretching were performed 3 times a week, totaling 10 sessions. Intensities were established according to the scale of perceived effort in flexibility. Passive and active knee extension ROM, passive peak torque (PPT), PPT angulation, viscoelastic stress-relaxation and modified shuttle run were measured at baseline, post 1st session (acute effect), and $48 \mathrm{~h}$ after the 10th session (chronic effect). Discomfort/pain and affective responses were recorded after each session and the end of training, respectively.

Results. No intergroup differences were observed after the 1st session. However, 48 $\mathrm{h}$ after the 10th session, the MDLS and PLS groups exhibited a similar significant increase in active and passive knee extension ROM compared to the NS $(\mathrm{P}<0.01$, effect size $=1.8$ to 2.6$)$ and CLS $(\mathrm{P}<0.01$, effect size $=1.5$ to 2.4$)$. There were no intergroup differences for the other outcomes.

Conclusions. Static stretching exercises do not need to be prescribed at pain-tolerated intensity for knee flexibility gains in amateur soccer players.
\end{abstract}

\section{KEY WORDS}

Hamstring muscles; joint flexibility; Muscle Stretching Exercises; torque; sports.

\section{BACKGROUND}

Adequate physical conditioning is essential for sports and muscle flexibility is a basic component of performance (1). In soccer, for instance, players need good flexibility to perform repeated high-intensity movements such as explosive sprints and powerful kicks (2). Shortening of multijoint lower limb muscles such as the hamstrings might cause muscle imbalance, which may lead to severe postur- al consequences and pain (3) in addition to compromising player performance (4).

Although recent studies have suggested that flexibility deficits are minor risk factors for hamstring injury (5) improving range of motion $(\mathrm{ROM})$ may influence the performance of soccer players $(6,7)$. After four weeks of static stretching (4 days/week), young elite soccer players showed an improvement in $35-\mathrm{m}$ speed, explosiveness and agility (6), while 7 weeks of static stretching (6 days/week) had a posi- 
tive effect on linear sprinting in a similar group (7). Thus, it is reasonable to assume that appropriate hamstring flexibility is important for training and rehabilitation protocols in this population.

Several parameters can elicit gains in joint flexibility (8), primarily stretch intensity, which represents the magnitude of force generated during the maneuver and may influence tissue response (8). To increase ROM, previous studies have suggested using higher intensity (i.e. stretching to the point of pain) rather than longer duration in clinical populations (9). However, soccer players are already subject to daily workloads (10) and high-intensity stretching to the point of pain may cause damage and, ultimately, be counter-productive (11). Thus, it may be beneficial to minimize overload during stretching as long as it remains effective.

From this perspective, low or high-intensity hamstring stretching may promote similar acute (one stretching session) gains in $\operatorname{ROM}(12,13)$. On the other hand, chronic training (repeated stretching for $>3$ weeks) (13) comparing different stretching intensities is scarce. A recent study found that long-term hamstring training by stretching at low or high-discomfort intensities promoted similar ROM gains in untrained men (14). In addition, no difference in flexibility was observed after static stretching training to the point of pain or discomfort in physically active women (11). No previous high-quality methodological studies were found that assessed the acute and chronic effects of different stretching intensities on the ROM and functional performance of soccer players. Given that stretching is often prescribed for these individuals, determining the minimum stretching intensity needed to generate effects on ROM will contribute to better flexibility training prescription. Moreover, passive torque, which is the total resistance of the muscle-joint complex during movement (15), perceived discomfort/pain and affective responses to stretching at different intensities have yet to be investigated in soccer players.

Thus, the main objective of this study was to investigate the acute and chronic effects of different static stretching intensities on the knee ROM, passive torque and functional performance of amateur soccer players. Discomfort/pain perception and affective responses to stretching at different intensities were also assessed. It was hypothesized that stretching to the point of mild discomfort would be sufficient to result in an acute and chronic increase in ROM compared to no stretching, stretching to the point of comfort or to the point of pain. In addition, passive torque and functional performance would not be altered, regardless of the intensity applied, and stretching to the point of pain would result in worse subjective perception outcomes.

\section{MATERIALS AND METHODS}

\section{Study design and ethical considerations}

This was a randomized clinical trial with concealed allocation and assessor blinding, carried out at the Neuromuscular Performance Analysis Laboratory. It was approved by the relevant ethics committee prior to data collection (number: 1.883.129) and conducted according to the guidelines of the Muscles, Ligaments and Tendons Journal (16) and Consolidated Standards of Reporting Trials (CONSORT). Before the study, the players were informed of the procedures and those who agreed to participate provided written consent. The trial was conducted according to the Helsinki Statement and prospectively registered at Clinical-Trials.gov (NCT03150563).

\section{Participants}

Forty competitive male soccer players were recruited through flyers, athletic trainers, coaches, and/or team physicians. A priori sample calculation was performed using $G^{*}$ Power (version 3.1; University of Trier, Trier, Germany) with previous published knee ROM data. Based on a previous study with 26 participants (11), $\alpha=0.05$, power $(1-\beta)=0.8$, correlation coefficient $=0.5$, and effect size $=0.28$ were adopted. According to these values, it was estimated that a total of 36 participants were needed. Considering a likely dropout rate of $15 \%, 40$ participants were targeted (10 per group). Individuals were included if they were aged $\geq 18$ years, engaged in soccer training sessions at least 3 times a week and had limited range of motion ( $15^{\circ}$ of active knee extension) (17). The ROM limitation of $15^{\circ}$ was needed because the players had to have the primary indication of stretching maneuvers. Potential players were excluded if they met any of the following criteria: acute injury or disorders that prevented them from undergoing the assessments or training sessions; prior lower limb or torso surgery; and taking drugs at the time of the evaluation that could cause muscle relaxation (17).

\section{Procedures}

All participants were randomly allocated (http://www. randomization.com/) to one of the following groups: no stretching (NS), comfort level stretching (CLS), mild discomfort level stretching (MDLS) and pain level stretching (PLS). The randomization process was conducted by an independent researcher (first researcher) who was not involved in other study procedures, using a computer-generated random numbers program. Each participant's random allocation was revealed just before the intervention. Another blind- 
ed researcher (second researcher) with clinical expertise in the musculoskeletal area assessed the dependent variables during the study period. The primary outcome was the active and passive knee extensions (AKE and PKE) evaluated with a goniometer. The secondary outcomes were passive torque measurements (passive peak torque (PPT), PPT angulation and viscoelastic stress-relaxation) obtained during an isokinetic dynamometer test. Viscoelastic stress-relaxation is the decline in passive tension over time when the muscle-tendon unit is held in a lengthened position (18). In addition, modified shuttle run (time in seconds), feeling of discomfort/pain (Visual Analogue Scale (VAS)) and affective valence (Feeling Scale) were measured.

The first evaluation was carried out at least 24 hours before (baseline) the start of the training or control period. Participants performed the tests in the following order: $\mathrm{ROM}$, passive torque and shuttle run test. ROM and passive peak torque measurements were performed with the non-dominant limb. The dominant limb was defined as the preferred leg used to kick a ball $(15,17)$. The participants assigned to the training groups (CLS, MDLS and PLS) participated in a hamstrings-targeted stretching program on both legs 3 times a week until completing 10 sessions. The following assessments occurred post $1^{\text {st }}$ session and $48 \mathrm{~h}$ after the $10^{\text {th }}$ session. Participants from the NS group underwent the same assessments, but did not undergo any intervention. During the training period, participants of all groups were asked to refrain from stretching, resistance and strength training outside the program.

\section{Outcome measures}

\section{Range of motion}

ROM was measured with the players in the supine position on a stretcher and their non-dominant hip on the support at $90^{\circ}$ flexion. The contralateral limb and pelvis were immobilized with a strap to avoid compensations (15). A goniometer (Carci ${ }^{\circledR}$; unit: degrees) was placed beside the leg and ROM recorded according to a previous study. Three AKE and PKE were performed, with a 30-second rest period between each trial. Maximum tolerable pain was used as the limit of movement for PKE. The average of the three repetitions was used for each outcome. Test-retest reliability was previously demonstrated to be excellent (ICC: 0.91-0.99) (19).

\section{Peak passive torque measurements}

Passive resistance to stretching was defined as the PPT of the hamstring muscle group during passive knee extension using an isokinetic dynamometer (Biodex Medical System 3 Pro, Shirley, NY, USA) at $5 \% \mathrm{~s}^{-1}$ (18). The dynamometer was calibrated before each assessment session. Participants sat on the dynamometer chair according to the manufacturer's instructions, with the hip flexed $110^{\circ}$. The axis of rotation was aligned with the lateral epicondyle, and the dynamometer lever arm fixed on the distal third of the subject's leg, approximately $5 \mathrm{~cm}$ above the lateral ankle malleolus. To avoid changes in position, participants were stabilized with straps in the pelvic and thoracic region, as well as on the thigh of the non-dominant limb. All passive torque measurements were gravity corrected.

The participants were asked to relax during the test while the non-dominant leg was passively extended. When they reached the maximum tolerable pain point, they pressed the 'stop' button, and the device recorded both PPT and PPT angulation. The player's leg was secured for 30 seconds in this position and the percentage $(\%)$ mean change ((final PPT : initial PPT) $\times 100$ ) was calculated to measure the viscoelastic stress-relaxation (18). This procedure was performed only once. Absence of active resistance during evaluation was determined through qualitative analysis of the PT chart. If any noise was detected within the graph, the test was repeated. The reliability of the PPT measurements was previously published, with ICC scores of 0.59 and 0.93 (18).

\section{Modified shuttle run test}

Functional analysis was carried out applying a modified 20-m sprint test, as previously described (17). This is a reliable (ICC: 0.99) (17), inexpensive and easy-to-perform test, with movements similar to those executed by soccer players, such as acceleration, deceleration, and change in direction. The subjects ran the first 10 meters as fast as possible, went around the cone $\left(180^{\circ}\right.$ change in direction on the side of the nondominant limb), and returned to the starting line (total $=20 \mathrm{~m}$ ). The players were familiarized with the test before undergoing 2 trials with 2-minute intervals between repetitions, and the fastest trial was used for data analysis.

\section{Feeling of discomfort/pain}

The subjective perception of discomfort/pain caused by passive stretching was recorded at the end of each session using the VAS (20). The scale consists of a 100-milimeter ruler, with "no discomfort/pain" $(0 \mathrm{~mm})$ written at one end and "maximum discomfort/pain" $(10 \mathrm{~mm})$ at the other. The average of the 10 sessions was used for analysis in each intervention group. VAS test-retest reliability has been shown to be high, with ICC scores of 0.88 and 0.92 (20).

\section{Affective valence}

The affective responses to training were determined using a Feeling Scale $(15,21)$, consisting of an 11-point bipolar scale ranging from +5 ("very good") to -5 ("very bad"). A previous study showed a strong correlation between the 
scale (r: 0.89-0.97) and ratings of perceived exertion (21). Participants indicated their affective relationship with the stretching program at the end of the intervention $(48 \mathrm{~h}$ after the $10^{\text {th }}$ session), according to the numbers on the scale.

\section{Stretching protocols}

A third researcher conducted lower limb stretch training on an individual basis. Passive static stretching protocols for hamstring muscles were performed with the player in the supine position on a stretcher (17). One limb in full extension was stabilized with a strap, while the other limb was stretched. Three 30-second sets of passive static stretching were carried out three times a week until completing 10 sessions, using the same rest period. The scale of perceived effort in flexibility (PERFLEX) was used to establish the sensation limits at each intensity (22). This scale consists of five stages of sensations for the different intensities, each referring to a level of ROM. Players from the experimental groups underwent the same protocols, differing according to stretching intensity: the CLS group performed each maneuver at the "forcing" intensity (31-60 on PERFLEX); the MDLS group stretched at the "discomfort" intensity (61-80 on PERFLEX); and the PLS group stretched at "bearable pain" (81-90 on PERFLEX). In order to establish the sensation for each group and determine the limit for each level, the limb was stretched to the intensity level immediately above the one previously established for the group, and then moved to the desired sensation limit. Before the start of the protocol, all the players were instructed on the correct use of the scale during stretching.

\section{Statistical analyses}

A fourth researcher who was not involved in the study conducted all analyses using commercial software. A parametric test was applied given that all the data were normally distributed (Kolmogorov-Smirnorv test), exhibiting homogeneous variances (Levene test). A two-way mixed-model ANOVA was performed for ROM, peak torque measure- ments and the modified shuttle run, with group (NS, CLS, MDSL and PLS) and time (before and after the $1^{\text {st }}$ session, and $48 \mathrm{~h}$ after the $10^{\text {th }}$ session) interaction. One-way ANOVA was carried out to compare intergroup sensation of discomfort/pain and affective valence. Tukey's test was used for post-hoc analysis to identify possible differences. The magnitude of the differences was calculated by testing Cohen's $d$. Significance was set at $5 \%$ for all analyses and the data expressed as mean \pm SD. Data were analyzed using the Statistical Package for the Social Sciences (SPSS; Chicago, IL, USA, Version 22.0).

\section{RESULTS}

Demographic characteristics of participants at baseline were similar for the four groups (table I). A total of 51 participants were screened and 9 were excluded for the reasons described in figure 1. Thus, 42 players underwent the $1^{\text {st }}$ intervention and were assessed immediately afterwards. One player from the PLS group was excluded due to injury and 41 participants completed all interventions (figure 1).

\section{Post $1^{\text {st }}$ session}

Intragroup analysis showed that athletes from all groups obtained a statistically significant increase in active and passive knee extension ROM ( $\mathrm{p}<0.05)$; however, only the PLS group exhibited a large effect size $\left(E S=1.3 ; \Delta=7.4^{\circ}(2.1,12.6)\right)$ for passive knee extension post $1^{\text {st }}$ intervention (table II). Moreover, PPT $(\mathrm{ES}=0.4)$ and PPT angle $(\mathrm{ES}=0.7)$ displayed a statistically significant increase in the MDLS group and PT angle rose in the CLS ( $E S=0.2)$ group after a single static stretching session $(\mathrm{P}<0.05$, table II). Intergroup analysis revealed no significant difference post $1^{\text {st }}$ session for any of the outcomes $(\mathrm{P}>0.05$, table III). However, there was a large effect size for active $\left(E S=0.9 ; \Delta=5.0^{\circ}(-2.6,12.7)\right)$ and passive $\left(\mathrm{ES}=1.2 ; \Delta=7.3^{\circ}(-0.7,15.3)\right)$ knee extension ROM in the PLS group compared to their NS counterparts (table III), and for viscoelastic stress-relaxation in the MDLS compared to the CLS group $(\mathrm{ES}=1.0 ; \Delta=6.5 \%(-0.9,13.9))($ table III).

Table I. Demographic data.

\begin{tabular}{|c|c|c|c|c|}
\hline & NS $(n=10)$ & $\operatorname{CLS}(n=11)$ & $\operatorname{MDLS}(n=10)$ & $\operatorname{PLS}(n=10)$ \\
\hline Age, mean (SD), years & $23.8 \pm 4.1$ & $24.7 \pm 4.8$ & $24.7 \pm 4.8$ & $22.8 \pm 2.1$ \\
\hline Weight, mean (SD), kg & $76.9 \pm 12.2$ & $79.2 \pm 8.9$ & $79.2 \pm 8.9$ & $72.6 \pm 9.3$ \\
\hline Height, mean (SD), cm & $1.8 \pm 0.1$ & $1.8 \pm 0.1$ & $1.8 \pm 0.1$ & $1.8 \pm 0.1$ \\
\hline $\begin{array}{l}\text { Body Mass Index, mean } \\
\text { (SD), } \mathrm{kg} / \mathrm{m}^{2}\end{array}$ & $23.9 \pm 2.8$ & $24.7 \pm 1.4$ & $24.7 \pm 1.4$ & $22.8 \pm 2.1$ \\
\hline
\end{tabular}

NS: no stretching; CLS, comfort level stretching; MDLS: mild discomfort level stretching; PLS: pain level stretching. 

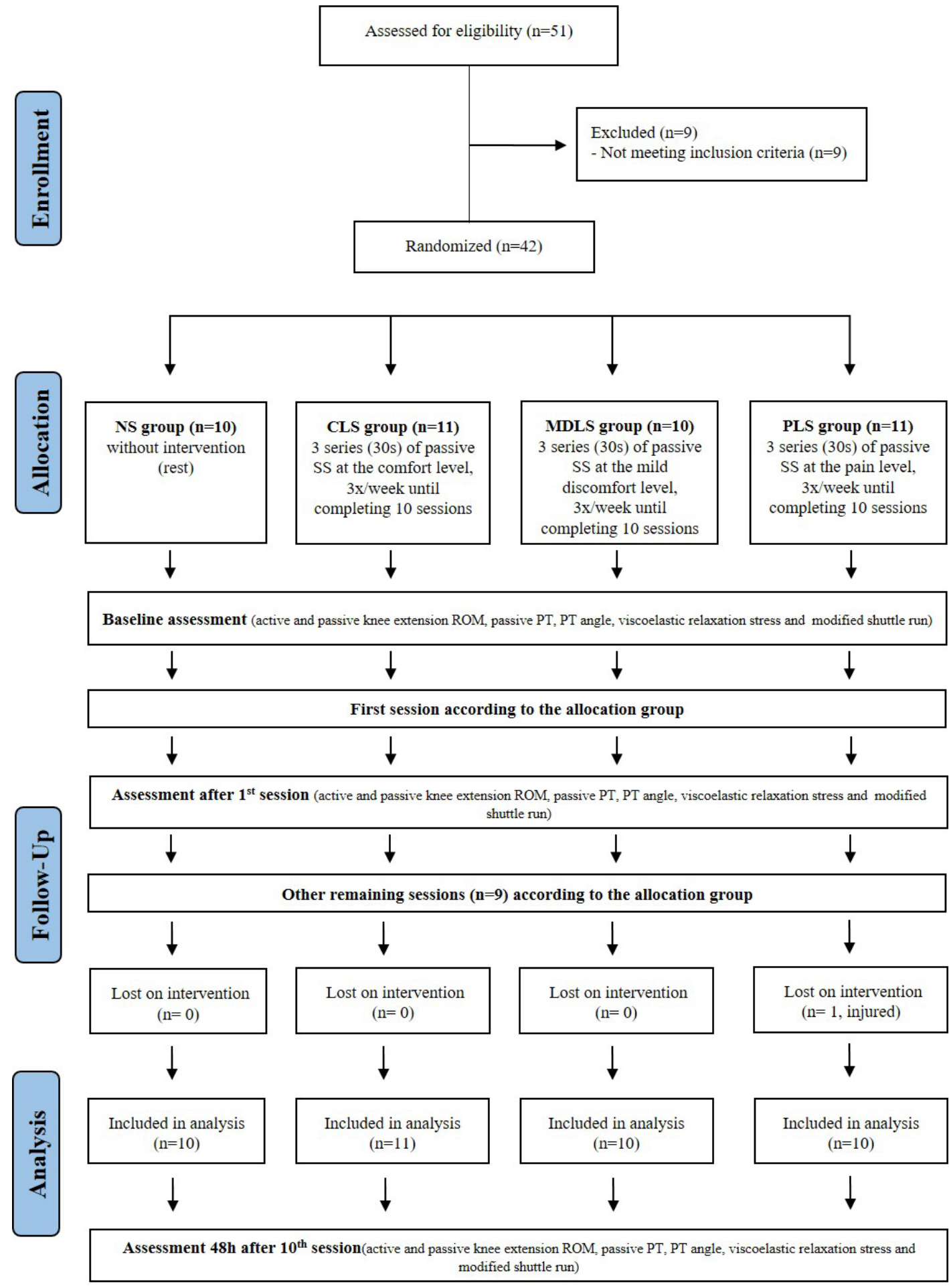

Figure 1. CONSORT flowchart. NS: no stretching;

CLS: comfort level stretching; MDLS: mild discomfort level stretching; PLS: pain level stretching; SS: static stretching; ROM: range of motion; PT: peak torque. 


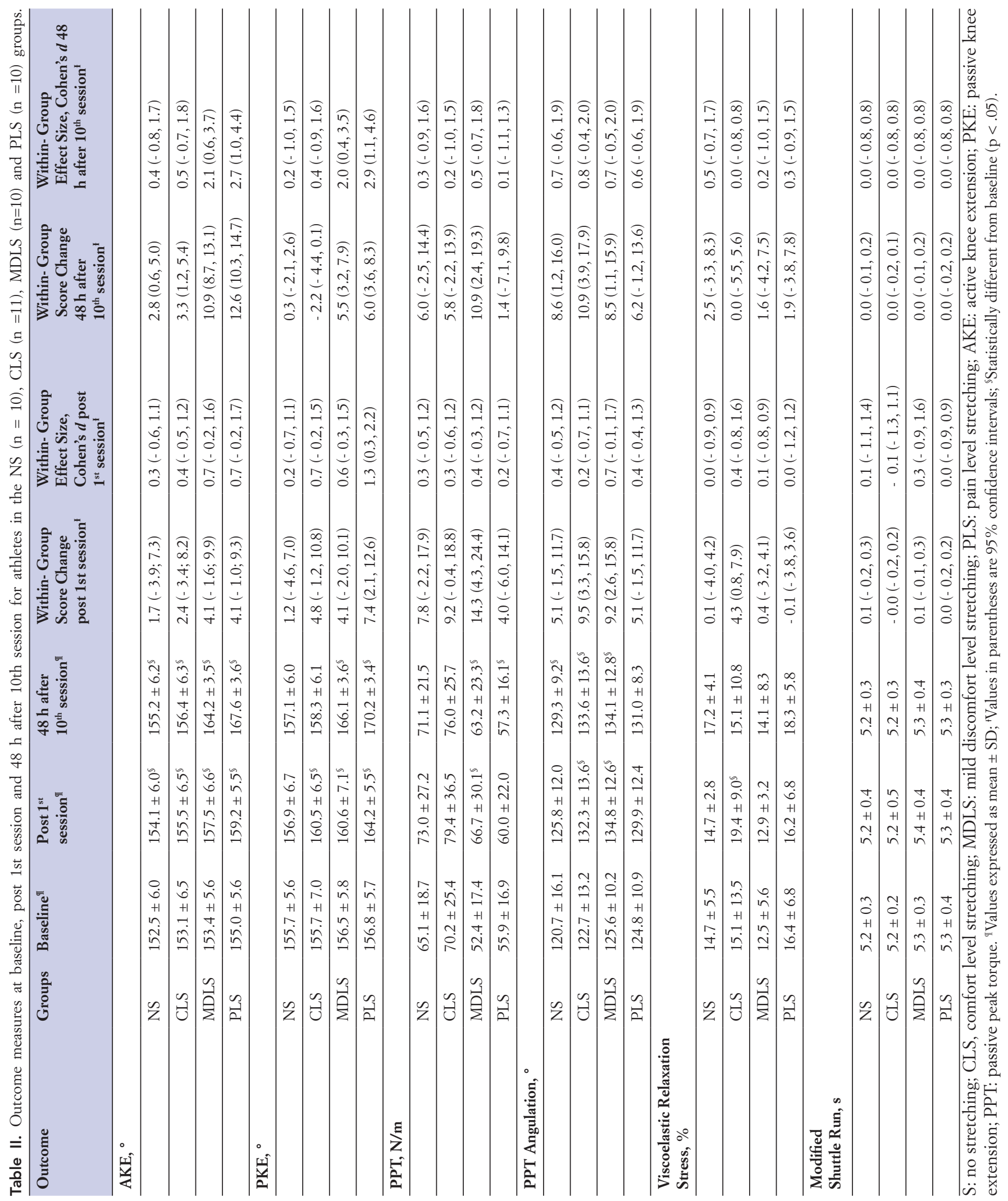




\section{$48 \mathrm{~h}$ after the $10^{\text {th }}$ session}

Intragroup analysis showed that athletes from all groups exhibited a statistically significant increase in active knee extension ROM, and only the PLS and MDLS groups increased passive knee extension ROM $(\mathrm{P}<0.05)$. A large effect size was observed for both outcome measures in the MDLS (active extension, $\mathrm{ES}=2.1, \Delta=10.9^{\circ}(8.7,13.1)$; passive extension, $\left.\mathrm{ES}=2.0, \Delta=5.5^{\circ}(3.2,7.9)\right)$ and PLS groups (active, $\mathrm{ES}=$ 2.1, $\Delta=12.6(10.3,14.7)$; passive, $\mathrm{ES}=2.9, \Delta=6.0(3.6,8.3))$ $48 \mathrm{~h}$ after the $10^{\text {th }}$ session (table II). Moreover, PPT showed a statistically significant increase in the MDLS $(\mathrm{ES}=0.5)$ and PLS $(E S=0.1)$ groups $(\mathrm{P}<0.05)$, and PPT angle in all the groups $(\mathrm{P}<0.05, \mathrm{ES}=0.7$ to 0.8$)$, except the PLS $(\mathrm{P}=0.10$, $\mathrm{ES}=0.6)($ table II). Intergroup analysis demonstrated a rise in active and passive knee extension $48 \mathrm{~h}$ after the $10^{\text {th }}$ session $(\mathrm{P} \leq 0.0001)$. Planned pairwise comparisons showed that the MDLS and PLS groups had similar $(\mathrm{P}>0.05)$ and significant increases in active and passive knee extension ROM compared to the NS $(\mathrm{P}<0.01, \mathrm{ES}=1.8$ to 2.6$)$ and $\mathrm{CLS}$ groups $(\mathrm{P}<$ 0.01 , ES $=1.5$ to 2.4$)$. In addition, the MDLS group experienced a large increase in both $\mathrm{ROM}$ variables compared to CLS participants $(\mathrm{P}<0.01 ; \mathrm{ES}=1.5)$ (table III).

Figure 2 shows the individual data and the change $48 \mathrm{~h}$ after the $10^{\text {th }}$ session in relation to baseline values for active (figure $2 \mathrm{~A}$ ) and passive (figure $2 \mathrm{~B}$ ) knee extension ROM in all the groups. There were no intergroup differences for any of the other outcomes after 10 sessions of passive static stretching $(\mathrm{P}>0.05$, table III).

\section{VAS score and affective valence}

The mean VAS score was different for the intervention groups $(\mathrm{p} \leq 0.0001)$. The PLS group obtained the highest scores $(79.6 \pm 8.4 \mathrm{~mm})$, followed by their MDLS $(64.7 \pm 12.5$ $\mathrm{mm})$ and CLS counterparts $(34.2 \pm 20.1 \mathrm{~mm}$, table IV). Affective valence showed similar $(\mathrm{P}>0.05)$ and higher $(\mathrm{p} \leq$ $0.001)$ positive responses for the PLS $(3.4 \pm 1.4)$ and MDLS $(3.4 \pm 1.2)$ compared to the CLS group $(0.3 \pm 1.6)$ after the static stretching protocol (table IV).

\section{DISCUSSION}

This is the first study to investigate the acute and chronic effects of different static stretching intensities on the knee ROM, passive torque and functional performance of amateur soccer players. The main finding was that passive static stretching training at the point of mild discomfort or pain, but not to the point of comfort, resulted in significant active and passive ROM improvements. Our results underscore that athletes do not need to experience pain during stretching maneuvers, since stretching with mild discomfort was sufficient to improve hamstring flexibility, with high magnitude effect sizes.

Our findings agree with previous studies conducted in different populations. In physically active women, a 4-week static and active hamstring stretching program (4 days/ week) to the point of pain or discomfort (according to the PERFLEX scale) showed a similar increase in hip flexion ROM (11). In another study, 12 weeks of hamstring stretching (3 days/week) at low or high discomfort (1-2 and 9-10 on the Verbal Numerical Scale, respectively) promoted similar active extension ROM gains in untrained men (14). In these studies, improved flexibility was attributed to the subjects' increased tolerance to stretching, since passive torque (i.e., muscle-tendon length) did not change after the protocols.
A

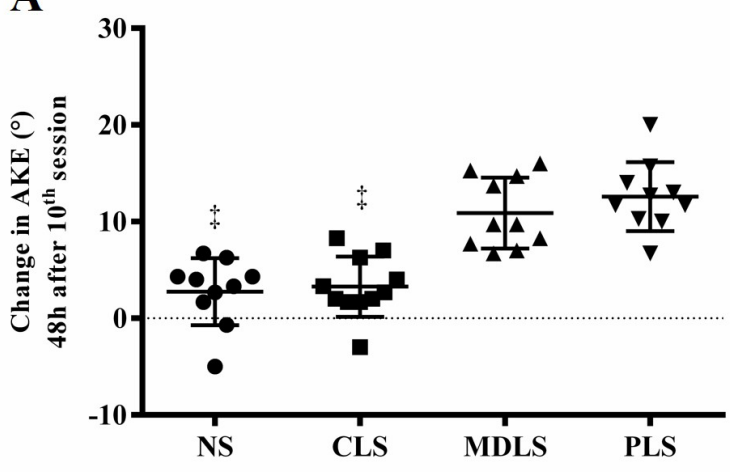

B

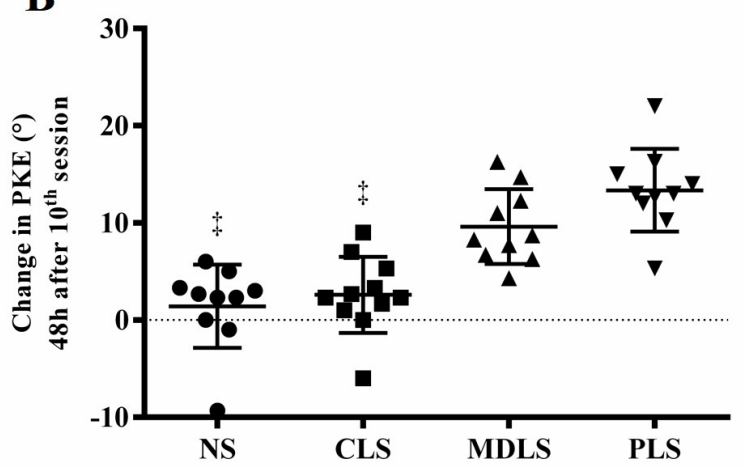

Figure 2. Individual data showing the change $48 \mathrm{~h}$ after the 10 th session in relation to baseline (mean \pm standard deviation) for active (A) and passive (B) knee extension (AKE and PKE, respectively) ROM in all groups. NS: no stretching; CLS: comfort level stretching; MDLS: mild discomfort level stretching; PLS: pain level stretching. ${ }^{*} \mathrm{p}<0.01 \mathrm{NS}$ or CLS groups $v s$ MDLS and PLS groups. 


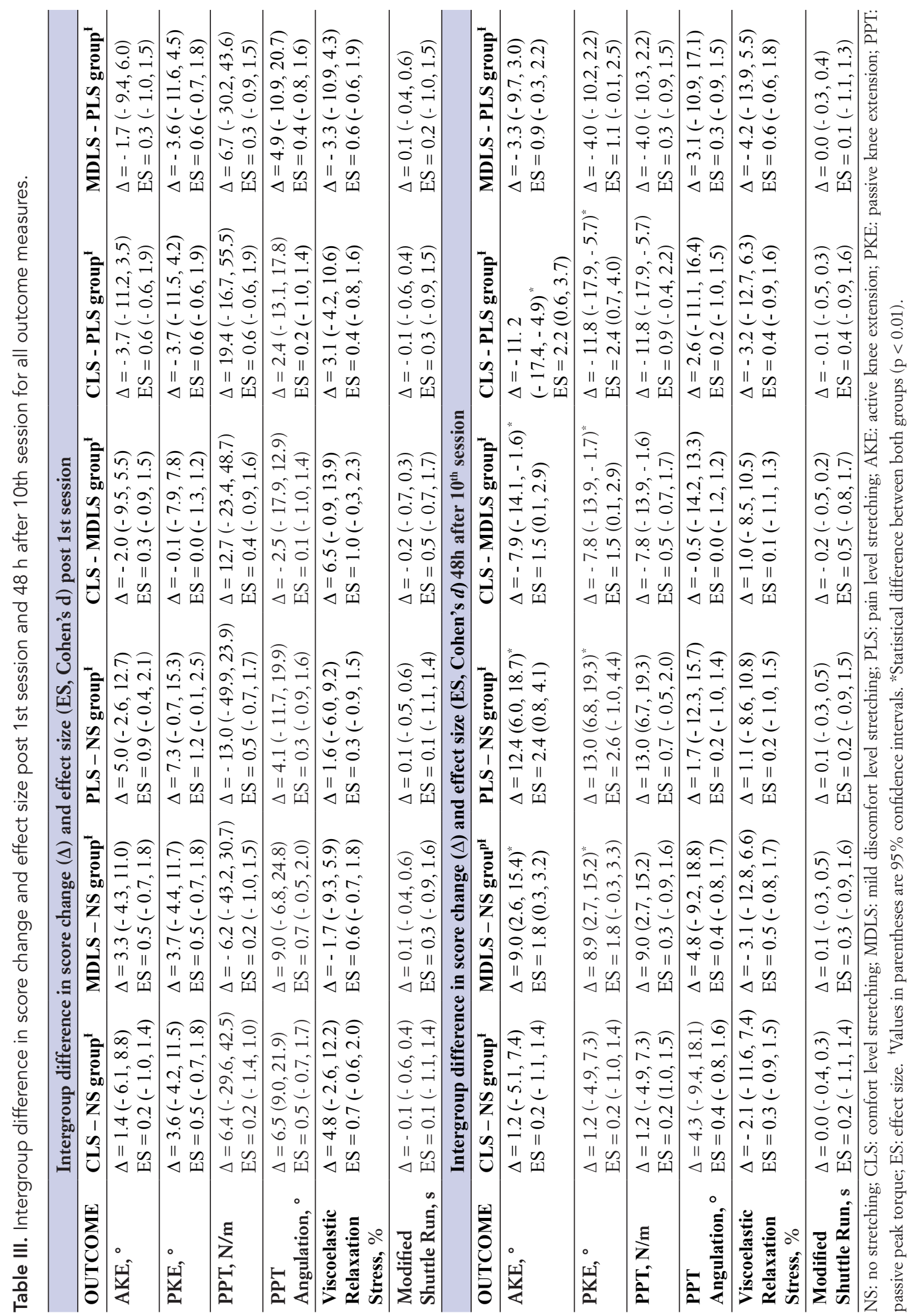


Table IV. Visual analogue scale and affective valence for experimental groups.

\begin{tabular}{|c|c|c|c|}
\hline OUTCOME & $\mathrm{CLS}^{\mathrm{T}}$ & MDLS $^{\text {T }}$ & PLS $^{\pi}$ \\
\hline VAS score, 0-100 mm & $34.2 \pm 20.1$ & $64.7 \pm 12.5$ & $79.6 \pm 8.4$ \\
\hline Affective Valence, $+5,-5$ & $0.3 \pm 1.6^{\#}$ & $3.4 \pm 1.2$ & $3.4 \pm 1.4$ \\
\hline OUTCOME & MDLS - CLS group ${ }^{\dagger}$ & PLS - CLS group ${ }^{\dagger}$ & PLS - MDLS group ${ }^{\dagger}$ \\
\hline VAS score, 0-100 mm & $\begin{array}{l}\Delta=30.5(11.6,49.4)^{*} \\
\mathrm{ES}=1.5(0.5,2.4)\end{array}$ & $\begin{array}{l}\Delta=45.4(30.9,59.9)^{*} \\
\mathrm{ES}=2.9(1.58,4.1)\end{array}$ & $\begin{array}{l}\Delta=14.9(0.4,29.4)^{*} \\
\mathrm{ES}=1.0(0.0,1.8)\end{array}$ \\
\hline
\end{tabular}

CLS: comfort level stretching; MDLS: mild discomfort level stretching; PLS: pain level stretching; VAS: visual analogue scale. "Values expressed as mean \pm SD. 'Values in parentheses are $95 \%$ confidence intervals. "Statistical difference between CLS and MDLS or PLS groups $(\mathrm{P}<0.05)$. *Statistical difference between both groups $(\mathrm{p} \leq 0.00)$.

Given that passive torque measurements (PPT, PPT angulation and viscoelastic stress-relaxation) were unchanged in our study, the ROM improvement was likely mediated by increased player tolerance to stretching, even at mild discomfort intensity. Several mechanisms are believed to be responsible for adapted tolerance, such as mechanoreceptor stimulation during the stretching maneuver, thereby inhibiting nociceptive signal transmission, and adaptation of nociceptive nerve endings, which play a role via neurotransmitter modulation or the gate control theory $(8,23)$.

By contrast, some authors have reported a decrease in PPT, and resulting changes in the torque-angle curve, after a static stretching regime $(24,25)$. These discrepant results may be due to the different parameters used in the studies. According to a recent review, higher average total stretching time (i.e., 1880s/week) is an important factor in decreasing passive joint stiffness (23). Furthermore, it appears that high stretching intensities associated with an extended program (in weeks) reduce passive stiffness. In healthy students, an 8 -week high-intensity static stretching protocol (5 days/ week, $1 \times 450 \mathrm{~s}$ ), more than five times that adopted in the present study, increased the fascicle length of knee flexors (25). In another study, a decrease in viscoelastic stress-relaxation was observed after 10-weeks of high-intensity (70-75\% torque $_{\max }$ ) static hamstring stretching (4 days/week, $6 \times 30$ s) in adult men (24). Although different stretching intensities were tested in the present study, the frequency and duration may not have been sufficient to affect connective tissue structures. Moreover, it is reasonable to assume that playing soccer may lead to hamstring muscle adaptations (i.e., musculotendinous stiffness), which may influence the response of connective tissue to stretching.

With respect to acute effects, a single passive static stretching session did not significantly alter the aforementioned outcomes, regardless of the intensity used. Although it is known that an acute bout of static stretching can increase
ROM (12), the magnitude of this effect may be partly due to controllable variables, such as stretching intensity, duration, type and position (8). In this study, the last three aforementioned parameters remained fixed and only intensity varied among the intervention groups. Regardless of stretching intensity, there were no significant differences in acute ROM between the experimental groups and controls. However, there was a larger effect size for active and passive knee extension ROM in the PLS versus NS group after the $1^{\text {st }}$ session. Given that stiffness (i.e., passive torque measurements) did not decline in the groups, corroborating previous studies $(12,13)$, the change in ROM may also be due to greater load tolerance before the end of the stretch (i.e., stretch tolerance). On the other hand, although the expression "no pain, no gain" is prevalent among athletes when it comes to stretching exercise, there are reasons to be cautious about passive stretching to the limits of pain because of the risk of soft tissue damage. A previous study found that acute muscle stretching to $90 \%$ of maximum ROM (pain perception) significantly increases systemic inflammation when compared to 30 and $60 \%$ of maximum ROM (gentle stretch intensity). Similarly, higher-intensity stretching might cause some muscle damage, which may reflect in delayed onset muscle soreness (11). In our study, there was no evidence of impaired functional performance (i.e., modified $20 \mathrm{~m}$ sprint run test). However, since muscle tissue damage after stretching maneuvers was not our focus, we cannot advise amateur soccer players to stretch to the point of pain to improve acute ROM. Additionally, considering that sports performance is the main focus of players and our stretching protocols did not enhance sprinting time, the intervention studied here should not be recommended for this purpose. Previous studies have also obtained controversial results on the effects of static stretching on sprint performance in soccer players $(7,17)$. Clearly, further research is needed to establish the influence 
of static stretching intensity on force-generating capacity involving other skills and specific sport movements.

Finally, one of the possible tools for assessing pleasure/displeasure with stretching programs is the feeling scale $(14,15)$. In this study, amateur soccer players who stretched at mild discomfort and pain intensities did not differ in their affective responses. However, the acceptance of both protocols was higher than for stretching at the comfort level. By contrast, a recent trial in untrained men found no differences in affective responses when stretching maneuvers were performed at low or high discomfort intensities (14). Although the authors reported that the feeling of pleasure/displeasure in relation to the degree of stretching discomfort was not affected by intensity, this response may depend on the population under study. In amateur soccer players, stretching to comfort level intensity seems not to be the best strategy to achieve a better affective response and an increase in ROM.

The results of this study appear to be consistent because we used robust methods, such as true randomization, concealed allocation, assessor blinding and quantification of stretching intensity. Moreover, the strongest point of the study is the sample of amateur soccer players. No previous research has investigated the effects of different stretching intensities on $\mathrm{ROM}$ and functional performance in this group. The present investigation, however, has some limitations. For instance, our sample did not include female athletes, or different clinical conditions and sports, thereby precluding extrapolation of the results. It would also be interesting to monitor muscle damage after the stretching program, since functional performance does not reveal whether different stretching intensities can alter muscle tissue. Additionally, assessing passive lengthening using the same posture as the static stretching

\section{REFERENCES}

1. Mariscal SL, Garcia VS, Fernández-García JC, Sáez de Villarreal E. Acute Effects of Ballistic vs Passive Static Stretching Involved in A Pre-Match Warm-Up Regarding Vertical Jump and Linear Sprint Performance in Soccer Players. J Strength Cond Res 2018:35:147-53.

2. Avrillon S, Lacourpaille L, Hug F, et al. Hamstring muscle elasticity differs in specialized high-performance athletes. Scand J Med Sci Sport 2020;30:83-91.

3. Gordon R, Bloxham S. A Systematic Review of the Effects of Exercise and Physical Activity on Non-Specific Chronic Low Back Pain. Healthcare 2016;4:22

4. García-Pinillos F, Ruiz-Ariza A, Moreno del Castillo R, Latorre-Román P. Impact of limited hamstring flexibility on vertical jump, kicking speed, sprint, and agility in young football players. J Sports Sci 2015;33:1293-7.

5. van Dyk N, Farooq A, Bahr R, Witvrouw E. Hamstring and Ankle Flexibility Deficits Are Weak Risk Factors for Hamstring Injury in Professional Soccer Players: A Prospective Cohort intervention, as well as monitoring hamstring electromyographic activity with a view to ensuring muscle relaxation during the evaluation of passive torque, should be considered in future studies. Finally, given that responses to stretching may occur primarily due to increased load tolerance (8), we suggest that future research investigate stretching protocols that more closely reflect the athlete's reality, with periodic load adjustments (i.e., variations in stretching intensity in the same group), as opposed to only testing protocols with fixed training variables during the entire program.

\section{CONCLUSIONS}

Static stretching exercises do not need to be prescribed at pain-tolerated intensity for knee flexibility gains in amateur soccer players. Mild discomfort level stretching is effective for increasing ROM and does not compromise functional performance. From a practical point of view, this information can help athletes, coaches and clinicians prescribe stretching in a more targeted manner.

\section{ACKNOWLEDGEMENTS}

The authors would like to gratefully acknowledge the participants who dedicated their time to the development of this study. The authors are also grateful to the Coordination for higher Education Staff Development (CAPES) for their financial support of this research.

\section{CONFLICT OF INTERESTS}

The authors declare that they have no conflict of interests.

Study of 438 Players Including 78 Injuries. Am J Sports Med 2018;46:2203-10.

6. Hadjicharalambous M. The effects of regular supplementary flexibility training on physical fitness performance of young high-level soccer players. J Sports Med Phys Fitness 2016;56:699-708.

7. Rodriguez Fernandez A, Sanchez J, Rodriguez-Marroyo JA, Villa JG. Effects of seven weeks of static hamstring stretching on flexibility and sprint performance in young soccer players according to their playing position. J Sports Med Phys Fitness 2016;56:345-51.

8. Apostolopoulos N, Metsios GS, Flouris AD, Koutedakis Y, Wyon MA. The relevance of stretch intensity and position-a systematic review. Front Psychol 2015;6:1128.

9. Dempsey AL, Branch TP, Mills T, Karsch RM. High-intensity mechanical therapy for loss of knee extension for worker's compensation and non-compensation patients. Sport Med Arthrosc Rehabil Ther Technol 2010;29:904-12. 
10. McCall A, Dupont G, Ekstrand J. Injury prevention strategies, coach compliance and player adherence of 33 of the UEFA Elite Club Injury Study teams: A survey of teams' head medical officers. Br J Sports Med 2016;50:725-30.

11. Muanjai P, Jones DA, Mickevicius M, Satkunskiene D, Snieckus A, Rutkauskaite R, et al. The effects of 4 weeks stretching training to the point of pain on flexibility and muscle tendon unit properties. Eur J Appl Physiol 2017;117:1713-25.

12. Santos CX, Beltrão NB, Pirauá ALT, Durigan JLQ, Behm D, de Araújo RC. Static Stretching Intensity Does Not Influence Acute Range of Motion, Passive Torque, and Muscle Architecture. J Sport Rehabil 2018;29:1-6.

13. Konrad A, Tilp M. Increased range of motion after static stretching is not due to changes in muscle and tendon structures. Clin Biomech 2014;29:636-42.

14. Beltrão NB, Ximenes Santos C, de Oliveira VMA, Pirauá ALT, Behm D, Pitangui ACR, et al. Effects of a 12-Week Chronic Stretch Training Program at Different Intensities on Joint and Muscle Mechanical Responses: A Randomized Clinical Trial. J Sport Rehabil 2019:1-9.

15. Barbosa GM, Figueirêdo Dantas GA, Silva BR, Souza TO, Brito Vieira WH. Static or dynamic stretching program does not change the acute responses of neuromuscular and functional performance in healthy subjects: a single-blind randomized controlled trial. Rev Bras Ciencias Do Esporte 2018;40:418-26.

16. Padulo J, Oliva F, Frizziero A, Maffulli N. Muscles, Ligaments and Tendons Journal - Basic principles and recommendations in clinical and field Science Research: 2018 update. Muscles Ligaments Tendons J 2018;8(3):305-7.

17. Barbosa GM, Trajano GS, Dantas GAF, Silva BR, Vieira WHB. Chronic Effects of Static and Dynamic Stretching on Hamstrings Eccentric Strength and Functional Performance: A Randomized Controlled Trial. J Strength Cond Res 2020;34:2031-9.

18. Beltrão NB, Santos CX, De Oliveira VMA, Pirauá ALT, Pitangui ACR, De Araújo RC. Test-retest reliability of the range of motion and stiffness based on discomfort perception. Isokinet Exerc Sci 2017;25:187-92.

19. Barbosa GM, Dos Santos HH, De Figueirêdo Dantas GA, Da Silva BR, Pinheiro SM, De Brito Vieira WH. Intra-rater and inter-instrument reliability on range of movement of active Knee extension. Motriz Rev Educ Fis 2017;23:53-9.

20. Badia Llach X, Monserrat S, Roset M, Herdman M. Feasibility, validity and test-retest reliability of scaling methods for health states: The visual analogue scale and the time trade-off. Qual Life Res 1999;8:303-10.

21. Rose EA, Parfitt G. Can the feeling scale be used to regulate exercise intensity? Med Sci Sports Exerc 2008;40:1852-60.

22. Martin Dantas EH. Scale of perceived exertion in the flexibility (PERFLEX): a dimensionless tool to evaluate the intensity? Fit Perform J 2008;7:289-94.

23. Freitas SR, Mendes B, Le Sant G, Andrade RJ, Nordez A, Milanovic Z. Can chronic stretching change the muscle-tendon mechanical properties? A review. Scand J Med Sci Sport 2018;28:794-806.

24. Peixoto GH, Andrade AG, Menzel HJK, Araújo SRS, Pertence AEM, Chagas MH. Viscoelastic stress relaxation in the hamstrings before and after a 10-week stretching program. Muscle and Nerve 2015;51:761-4.

25. Freitas SR, Mil-Homens P. Effect of 8-week high-intensity stretching training on biceps femoris architecture. J Strength Cond Res 2015;29:1737-40. 